International Journal of Wireless \& Mobile Networks (IJWMN) Vol. 3, No. 4, August 2011

\title{
ANALYTIC STUDY OF SYNCHRONIZATION ERRORS IN OFDM SYSTEMS APPLIED ON WLAN TRANSCEIVER
}

\author{
Mourad MELLITI $^{1}$, Salem HASNAOUI ${ }^{1}$ and Ridha BOUALLEGUE ${ }^{2}$ \\ ${ }^{1}$ Department of Computer and Communication Technologies \\ National School of Engineering of Tunis (ENIT) \\ mourad.mellitidenit.rnu.tn, salem.hasnaouidenit.rnu.tn \\ ${ }^{2} 6$ 'Tel Research Unit, Higher School of Communications of Tunis, Sup'Com, Tunisia \\ ridha.boualleguedsupcom.rnu.tn
}

\begin{abstract}
In this paper, a new energetic approach was developed to study the impact of synchronization errors on OFDM transmission systems. Indeed, this approach can be applied in wireless communication systems such as HiperLAN and WLAN Transceivers. Firstly, the studies were conducted on the impact of timing errors on system performance and we have shown that stress recovery sampling frequency were particularly hard because of the use of a minimum number of pilot symbols and a maximum number of states of the constellations. The mathematical approach we followed in this paper which is an original contribution in the RF field can jointly estimate the channel response and the frequency offset. We realized, along with mathematical analysis, simulations to evaluate the performance of our approach. The results can evaluate the performance of the system against synchronization effects.
\end{abstract}

\section{Keywords}

OFDM, WLAN Transceivers, Frequency Offset, Synchronization effects.

\section{INTRODUCTION}

Several previous studies have investigated and presented general specifications of OFDM systems and identify RF synchronization objectives associated with them. For this reason, it appears that a detailed mathematical analysis of synchronization errors on transmission systems is unavoidable. Indeed, this paper outlines specific problems related to time and frequency offsets at the reception of the OFDM signal which is superimposed with phase noise from a local oscillator located in the receiver. Two phase error effects must be distinguished: interference between carriers and the common phase error. A mathematical analysis will be introduced in this paper to show the quantitative importance of these effects. Among the goals of this work is to demonstrate that the phase noise was not an obstacle in the implementation of a complete digital transmission system and it is essential to carefully evaluate the effects of synchronization errors caused by noise due to channel estimation errors, phase noise, frequency offset, the frame errors and the time lag in the design of receivers.

\section{Energetic Analysis of Errors on the Performance DEGRADATION OF OFDM SYSTEMS}

In the following paragraph we will analyze and quantify the various errors that may occur during a transmission OFDM applied to our architecture and caused by a non-ideal synchronization in the placement of the FFT window, the problems of synchronization clock, and the problems of channel estimation. For these studies we illustrate below some concepts to 
International Journal of Wireless \& Mobile Networks (IJWMN) Vol. 3, No. 4, August 2011

be introduced and some formulas that are necessary to be mentioned and that will be useful throughout the remainder of this paper. Consider the following report introduces the ideal relationship between the ideal SNR and the real SNR and recall that the actual signal to noise ratio means the quality of a transmission of information compared to parasites [1] [2] [3]:

$$
\Delta \gamma=\frac{\gamma_{\text {ideal }}}{\gamma_{\text {real }}}
$$

The signal to noise ratio in the case of a perfect synchronization can lead to the following relation while considering that the only disturbances are caused by a Gaussian channel:

$$
\gamma_{\text {idéal }}=\frac{E_{s}}{\sigma_{A W G N}^{2}}
$$

While the signal to noise ratio with a non-ideal synchronization is given by the following relation:

$$
\gamma_{\text {réel }}=\frac{E_{s}}{\sigma_{A W G N}^{2}+\sigma_{\varepsilon}^{2}\left(E_{s} ; \sigma_{A W G N}^{2}\right)}
$$

While:

$\Delta \gamma \quad$ SNR Loss due to non-ideal synchronization

$E_{s} \quad$ Averaged received symbol energy

$\sigma_{A W G N}^{2}$ Noise power (AWGN)

$\sigma_{\varepsilon}^{2} \quad$ Is the sum of the different sources of disturbance other than the AWGN as:

$$
\sigma_{\varepsilon}^{2}=\sigma_{h}^{2}+\sigma_{p h}^{2}+\sigma_{\Omega}^{2}+\sigma_{t}^{2}+\sigma_{f d}^{2}
$$

Where:

$\sigma_{h}^{2} \quad$ Due to channel estimation error

$\sigma_{p h}^{2} \quad$ Due to phase noise

$\sigma_{\Omega}^{2} \quad$ Due to frequency offsets

$\sigma_{t}^{2} \quad$ Due to frame and timing errors

$\sigma_{f d}^{2} \quad$ Due to fast fading

The report given by equation (3.1) can be reproduced by introducing the concepts of powers of the noise data distributed by equation (3.4) as follows:

$$
\Delta \gamma=\frac{\gamma_{\text {idéal }}}{\gamma_{\text {real }}}=\frac{\sigma_{A V G N}^{2}+\sigma_{\varepsilon}^{2}\left(E_{s} ; \sigma_{A W G N}^{2}\right)}{\sigma_{A V G N}^{2}}=1+\frac{\sigma_{\varepsilon}^{2}\left(E_{s} ; \sigma_{A V G N}^{2}\right)}{\sigma_{A V G N}^{2}}
$$

Naturally if we raise the ratio of SNR loss in case of bad timing by the maximum loss in SNR as follows:

$$
\Delta \gamma \leq(\Delta \gamma)_{\max }
$$


Then equation (3.5) leads us to increase the following report:

$$
\sigma_{\varepsilon}^{2}\left(E_{s} ; \sigma_{A V G N}^{2}\right) \leq \sigma_{A V G N}^{2} \cdot\left\{(\Delta \gamma)_{\max }-1\right\}
$$

\section{QUANTIFICATION OF SYNCHRONIZATION ERRORS ON THE SHIFT FRAMES}

Before addressing the various cases of lag on the arrival of frames at the receiver we will recall the waveform received with cyclic prefix, which can be written as a vector of symbols that is reflecting the simplified expression of the signal at the receiver input and resulting in the following simplified form [4] [6] [7]:

$$
y_{n}=\frac{1}{N} \sum_{k=-K}^{K} X_{k} H_{k} e^{\frac{j 2 \pi n k}{N}}
$$

After FFT processing the form of this vector of received symbols becomes:

$$
\begin{aligned}
& Y_{k}=\sum_{n=0}^{N-1} y_{n} e^{\frac{-j 2 \pi n k}{N}}=X_{k} H_{k} \\
& H_{k}=\sum_{n=0}^{N-1} h_{n} e^{\frac{-j 2 \pi n k}{N}}
\end{aligned}
$$

The time lag of one symbol is expressed by introducing a time error $\delta T_{S}$ in the entire duration of the symbol which becomes $\left(\varepsilon_{t}+\delta\right) T_{S}$ where $\varepsilon_{t}$ is an integer and $\delta$ belongs to $[0,1]$.

\subsection{Errors for a non-frequency selective channel}

Now treat the case where the channel is not frequency selective $\varepsilon_{t}>0$ as shown in Figure 1 below:

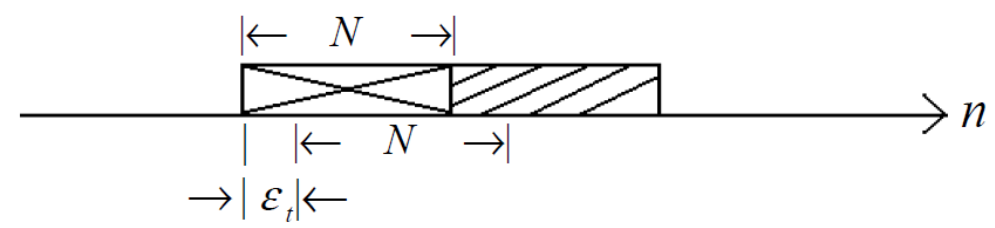

Figure 1. Cas d'un canal non sélectif en fréquence pour $\varepsilon_{t}>0$

We then define in the sequel $y_{l, n}$ as the $l^{\text {ieme }}$ block with the $n^{\text {ieme }}$ sample devoid of the noise caused by the phase shift.

$$
\begin{aligned}
Y_{l, k}= & \sum_{n=0}^{N-1-\varepsilon_{t}} y_{l, n+\varepsilon_{t}} e^{\frac{-j 2 \pi n k}{N}}+\sum_{n=N-\varepsilon_{t}}^{N-1} y_{l+1, n-\left(N-\varepsilon_{t}\right)} e^{\frac{-j 2 \pi n k}{N}} \\
Y_{l, k}= & \sum_{n=0}^{N-1-\varepsilon_{t}}\left(\frac{1}{N} \sum_{k=-K}^{K} X_{l, i} e^{\frac{j 2 \pi i\left(n+\varepsilon_{t}\right)}{N}}\right) e^{-j \frac{2 \pi n k}{N}} \\
& +\sum_{n=N-\varepsilon_{t}}^{N-1}\left(\frac{1}{N} \sum_{i=-K}^{K} X_{l+1, i} e^{\frac{j 2 \pi i\left(n-N+\varepsilon_{t}\right)}{N}}\right) e^{-j \frac{2 \pi n k}{N}}
\end{aligned}
$$




$$
\begin{aligned}
& Y_{l, k}=\overbrace{\frac{N-\varepsilon_{t}}{N} X_{l, k} e^{\frac{j 2 \pi k \varepsilon_{t}}{N}}}^{+} \overbrace{\frac{1}{N} \sum_{n=0}^{N-1-\varepsilon_{t}}\left(\sum_{i=-K, i \neq K}^{K} X_{l, i} e^{\frac{j 2 \pi\left(n+\varepsilon_{t}\right)}{N}}\right) e^{-j \frac{2 \pi n k k}{N}}}^{I C I} \\
& \underbrace{+\frac{1}{N} \sum_{n=N-\varepsilon_{t}}^{N-1}\left(\sum_{i=-K}^{K} X_{I+1, i} e^{\frac{j 2 \pi i\left(n-N+\varepsilon_{t}\right)}{N}}\right) e^{-j \frac{2 \pi m k}{N}}}_{\text {Part } 3}
\end{aligned}
$$

Where $\frac{N-\varepsilon_{t}}{N}$ in Part 1 is the coefficient showing the loss of signal and $e^{\frac{j 2 \pi k \varepsilon_{t}}{N}}$ is the phase shift term. So the quantization errors on the time lag expressed by calculating the variance of the last two terms Part 2 and Part 3 of the previous relation :

$$
\sigma_{t}^{2}\left(E_{s} ; K ; N ; k\right)=V A R\left[\begin{array}{l}
\frac{1}{N} \sum_{n=0}^{N-1-\varepsilon_{i}}\left(\sum_{i=-K, i \neq K}^{K} X_{l, i} e^{\frac{j 2 \pi\left(n+\varepsilon_{t}\right)}{N}}\right) e^{-j \frac{2 \pi n k}{N}}+ \\
\frac{1}{N} \sum_{n=N-\varepsilon_{t}}^{N-1}\left(\sum_{i=-K}^{K} X_{l+1, i} e^{\frac{j 2 \pi i\left(n-N+\varepsilon_{t}\right)}{N}}\right) e^{-j \frac{2 m l k}{N}}
\end{array}\right]
$$

\subsection{Errors of a noiseless non-frequency selective channel}

In the case of a noiseless non-frequency selective channel we will rely on the assumptions of non-selectivity following:

$$
h(t, \tau)=h(t), \quad y_{n}=x_{n} h_{n}, \quad n=0 \cdots N-1, \quad h_{n}=\left.h(t)\right|_{t=n T_{s}}
$$

We can write the vector of received symbols as follows:

$$
Y_{k}=\sum_{n=0}^{N-1} x_{n} h_{n} e^{\frac{-j 2 \pi n k}{N}}
$$

We replace $x_{n}$ by its value, and then we can rewrite the vector of received symbols as follows:

$$
\begin{aligned}
Y_{k} & =\sum_{n=0}^{N-1} h_{n}\left[\frac{1}{N} \sum_{m=-K}^{K} X_{m} e^{\frac{-j 2 \pi m m}{N}}\right] e^{\frac{-j 2 \pi n k}{N}} \\
\text { Or: } \quad Y_{k} & =\sum_{m=-K}^{K} X_{m}\left[\frac{1}{N} \sum_{n=0}^{N-1} h_{n} e^{\frac{-j 2 \pi n(k-m)}{N}}\right]
\end{aligned}
$$

Otherwise in writing, the vector of received symbols can be separated into two components to make appeared the ICI noise related to the additive white Gaussian noise (AWGN) [1] [3] [9] [10] [11] [13].

Where

$$
Y_{k}=X_{k} \overline{h_{n}}+\overbrace{\sum_{m=-K, m \neq k}^{K} X_{m} \cdot H_{1, k-m}}^{I C I(A W G N)}
$$


International Journal of Wireless \& Mobile Networks (IJWMN) Vol. 3, No. 4, August 2011

Now energetically, we can simplify the power of the fast fading by calculating the variance of the noise component in the vector of received symbols given by the following relation:

$$
\sigma_{f d}^{2}\left(E_{s} ; K ; k ; h\right)=V A R\left[\sum_{m=-K, m \neq K}^{K} X_{m} \cdot H_{1, k-m}\right]
$$

\subsection{Errors of a noiseless frequency selective channel}

In this section we will develop equations for the effect of a noiseless frequency selective channel as in the previous paragraph we quantify the power of synchronization effects related to interference from the temporal selectivity.

Let the following assumptions:

$$
y_{n}=\sum_{l=0}^{L} x_{n-1} h_{n-l}, \quad n=0 \cdots N-1
$$

Where $h_{n, l}$ represent the channel response as $h_{n, l}=\left.h(t, \tau)\right|_{t=n T_{s}, \tau=l T_{s}}$ with the condition: $h(t, \tau)=0, \quad \tau \geq L T_{s}, \quad L \leq N_{g}$

$$
Y_{k}=\sum_{n=0}^{N-1} y_{n} e^{\frac{-j 2 \pi n k}{N}}
$$

However, according to the above assumptions and substituting its value is obtained:

$$
\begin{aligned}
& Y_{k}=\sum_{n=0}^{N-1}\left(\sum_{l=0}^{L} x_{n-l} h_{n, l}\right) e^{\frac{-j 2 \pi n k}{N}} \\
& Y_{k}=\sum_{n=0}^{N-1}\left[\sum_{l=0}^{L}\left(\frac{1}{N} \sum_{m=-K}^{K} X_{m} e^{\frac{-j 2 \pi(n-l) m}{N}}\right) h_{n, l}\right] e^{\frac{-j 2 \pi n k}{N}} \\
& Y_{k}=\sum_{m=-K}^{K} X_{m}\left[\sum_{l=0}^{L}\left(\frac{1}{N} \sum_{n=0}^{N} h_{n, l} e^{\frac{j 2 \pi n(m-k)}{N}}\right)\right] e^{\frac{-j 2 \pi l m}{N}}
\end{aligned}
$$

However, $h_{n, l}=h_{l} \Rightarrow \frac{1}{N} \sum_{n=0}^{N} h_{l} e^{\frac{j 2 \pi n(m-k)}{N}}=0, \quad m \neq k$

What makes the equation (24) as follows:

$$
\begin{array}{r}
Y_{k}=X_{k} \sum_{l=0}^{L} h_{l} e^{\frac{-j 2 \pi k}{N}}=X_{k} \cdot H_{k} \\
\text { However, } H(m, k)=\sum_{l=0}^{L}\left(\frac{1}{N} \sum_{n=0}^{N} h_{n, l} e^{\frac{j 2 \pi n(m-k)}{N}}\right) e^{\frac{-j 2 \pi t m}{N}}
\end{array}
$$

This gives a new expression vector symbols as: 


$$
Y_{k}=\sum_{m=-K}^{K} X_{m} \cdot H(m, k)
$$

However,

$$
H(m, k)=\sum_{n=0}^{N-1}\left(\frac{1}{N} \sum_{l=0}^{L} h_{n, l} e^{\frac{-j 2 \pi m m}{N}}\right) e^{\frac{j 2 \pi n(m-k)}{N}}
$$

And:

$$
H(k, k)=\sum_{l=0}^{L}\left(\frac{1}{N} \sum_{n=0}^{N} h_{n, l}\right) e^{\frac{-j 2 \pi k k}{N}}=\sum_{l=0}^{L} \bar{h}_{l} e^{\frac{-j 2 \pi k k}{N}}
$$

Where the final expression vector of received symbols:

$$
Y_{k}=X_{k} \cdot H(k, k)+\overbrace{\sum_{m=-K, m \neq k}^{K} X_{k} H(m, k)}^{I C I}
$$

Where the energy quantization of the ICI effects caused by selectivity $\sigma_{f d}^{2}$ :

$$
\sigma_{f d}^{2}\left(E_{s} ; K ; k ; h\right)=E\left[\left(\sum_{m=-K, m \neq K}^{K} X_{k} \cdot H(m, k)\right)^{2}\right]
$$

\section{QUANTIFYING OF THE LOCAL OSCILLATOR ERRORS}

In practice, we use oscillators that are subject to phase noise, a random perturbation of the sine wave phase stationary [7] [12] [14]. Since we will keep the assumption that uses modules of the FFT and IFFT for modulation and demodulation of the OFDM signal and to introduce the notion of error, we will resume the received signal with a phase noise and frequency offset is:

$$
y(t)=\sum_{k=0}^{N-1} x_{k} H_{k}(t) e^{j\left(2 \pi f_{o} t+\theta(t)\right)}
$$

The carrier can be developed with a frequency offset and phase noise as follows:

$$
e^{j\left(2 \pi f_{o} t+\theta(t)\right)}, \quad \theta(t)=2 \pi \delta_{f} t+\varphi(t)
$$

Where $f_{\Delta}$ characterizes the frequency offset and $\varphi(t)$ characterizes the phase noise when $|\varphi(t)| \ll 2 \pi$ all assuming that the disturbances caused by the random process are relatively small. Then we can write:

$$
e^{j \varphi(t)} \approx 1+j \varphi(t)
$$

Where the carrier will have the following form:

$$
e^{j\left(2 \pi f_{0} t+\theta(t)\right)} \approx e^{j 2 \pi\left(f_{0}+\delta_{\mathrm{f}}\right) t}(1+j \varphi(t))
$$


Where $f_{\Delta}$ and $\varphi(t)$ characterize the overall effects of the oscillators of the transmitter and receiver $\delta_{\mathrm{f}}$ characterized by the use of ppm (part per million) for example: if $f_{0}=2,4 \mathrm{GHz}$ then $\pm 3 \mathrm{ppm} \rightarrow \pm 6,8 \mathrm{KHz}$

$\varphi_{t)}$ Is a stationary process broadly characterized by a spectral density function such as:

$$
S_{\varphi}(f)=\frac{F\left\{R_{\varphi}(\tau)\right\}}{C}
$$

With $R_{\varphi}(\tau)=E[\varphi(t) \varphi(t+\tau)]$ Where $\mathrm{C}$ : is the carrier power, $\mathrm{F}\{$.$\} is the Fourier transform and$ $\mathrm{E}$ [.] is the expectation which the shape of the spectral density power oscillator types is illustrated in Figure 2. According to the model proposed in [34] we have:

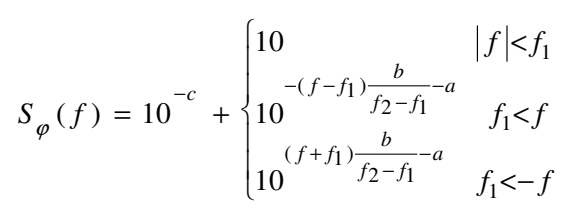

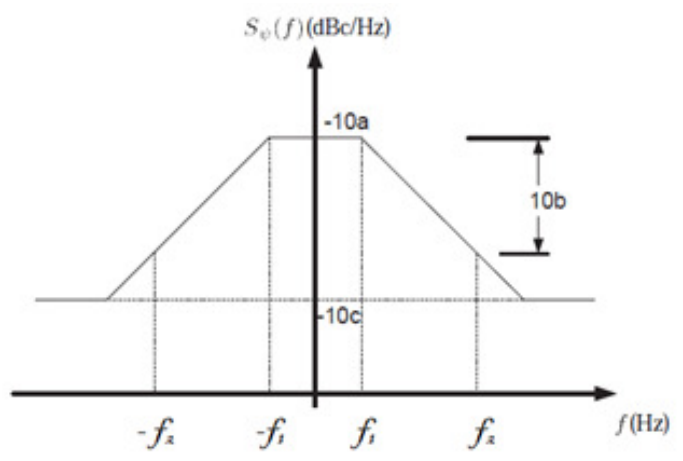

Figure 2. PSD of phase noise in a typical oscillator.

Typical values are: $a=6.5, \quad b=4, c=10.5 f_{1}=1 \mathrm{KHz}, f_{2}=10 \mathrm{KHz}$ Where $c$ is the background

\section{QUANTIFICATION OF FREQUENCY OFFSET ERRORS}

The OFDM systems are very sensitive to frequency shifts. In Indeed, if such shifts are not compensated, the subcarriers lose their orthogonality and receiver performances are thereby degraded[15] [16] [17] [18]. Quantization errors due to degradation performance due to a frequency offset is developed in following paragraphs.

The components of the vector of received symbols are of the form:

$$
Y_{k}=\frac{1}{N} \sum_{n=0}^{N-1} y_{n} e^{\frac{j 2 \pi\left(k-\varepsilon_{\Omega}\right) n}{N}}, \text { when } \delta_{f}=-\varepsilon_{\Omega} \frac{1}{N T_{S}}
$$

By replacing $y_{n}$ by its expression can then rewrite the vector of received symbols as follows: 
International Journal of Wireless \& Mobile Networks (IJWMN) Vol. 3, No. 4, August 2011

$$
Y_{k}=\sum_{n=0}^{N-1}\left[\sum_{l=0}^{L}\left(\frac{1}{N} \sum_{m=-K}^{X_{n-1}} X_{m} e^{\frac{j 2 \pi(n-1) m}{N}}\right) h_{l}\right] e^{\frac{-j 2 \pi\left(k-\varepsilon_{\Omega)} n\right.}{N}}
$$

This can also be written:

$$
Y_{k}=\sum_{m=-K}^{K} X_{m}\left(\sum_{l=0}^{L} h_{l} e^{\frac{-j 2 \pi l m}{N}}\right) \cdot \frac{1}{N} \sum_{n=0}^{N-1} e^{\frac{j 2 \pi\left(m-k+\varepsilon_{\Omega)} n\right.}{N}}
$$

This gives:

$$
Y_{k}=\sum_{m=-K}^{K} X_{m} H_{m} \cdot \frac{1}{N} \sum_{n=0}^{N-1} e^{\frac{j 2 \pi\left(m-k+\varepsilon_{\Omega)} n\right.}{N}}
$$

Writing that can be analyzed according to the value of $\varepsilon_{\Omega}$

Where $\varepsilon_{\Omega}$ is an integer then we have:

$$
\frac{1}{N} \sum_{n=0}^{N-1} e^{\frac{j 2 \pi\left(m-k+\varepsilon_{\Omega)} n\right.}{N}}=\left\{\begin{array}{lc}
1 & m+\varepsilon_{\Omega}=k \\
0 & \text { sinon }
\end{array}\right.
$$

Which gives:

$$
Y_{k}=X_{k-\varepsilon_{\Omega}} H_{k-\varepsilon_{\Omega}}
$$

Where $\varepsilon_{\Omega}$ is not an integer then we have:

$$
Y_{k}=X_{k} H_{k} \frac{1}{N} \sum_{n=0}^{N-1} e^{\frac{j 2 \pi m \varepsilon_{\Omega}}{N}}+\frac{1}{N} \sum_{m=-K, m \neq k}^{N-1} X_{m} H_{m}\left(\sum_{n=0}^{N-1} e^{\frac{j 2 \pi n\left(m+\varepsilon_{\Omega}-k\right)}{N}}\right)
$$

By mathematical simplification we can write:

$$
\frac{1}{N} \sum_{n=0}^{N-1} e^{\frac{j 2 \pi n \varepsilon_{\Omega}}{N}}=\frac{1}{N} \frac{1-e^{j 2 \pi \varepsilon_{\Omega}}}{1-e^{\frac{j 2 \pi \varepsilon_{\Omega}}{N}}}
$$

This can also be written in another way:

$$
\frac{1}{N} \sum_{n=0}^{N-1} e^{\frac{j 2 \pi n \varepsilon_{\Omega}}{N}}=\frac{\sin \pi \varepsilon_{\Omega}}{N \sin \frac{\pi \varepsilon_{\Omega}}{N}} e^{j \pi \frac{(N-1) \varepsilon_{\Omega}}{N}}
$$

After change of variables we can write: 
International Journal of Wireless \& Mobile Networks (IJWMN) Vol. 3, No. 4, August 2011

$$
\frac{1}{N} \sum_{n=0}^{N-1} e^{\frac{j 2 \pi n\left(m+\varepsilon_{\Omega}-k\right)}{N}}=\frac{\sin \pi\left(m+\varepsilon_{\Omega}-k\right)}{N \sin \frac{\pi\left(m+\varepsilon_{\Omega}-k\right)}{N}} e^{j \pi \frac{(N-1)\left(m+\varepsilon_{\Omega}-k\right)}{N}}
$$

We can write the increases relationship as follows:

$$
\frac{1}{N} \sum_{n=0}^{N-1} e^{\frac{j 2 \pi n\left(m+\varepsilon_{\Omega}-k\right)}{N}}<\frac{1}{N \sin \frac{\pi\left(m+\varepsilon_{\Omega}-k\right)}{N}} e^{j \pi \frac{(N-1)\left(m+\varepsilon_{\Omega}-k\right)}{N}}
$$

Based on the following assumptions and introducing the concept of energy per symbol and always under the assumption of independent white symbols having the same energy, we get[1] [2] [7] [8] [11] [15] [19]:

$$
E\left[X_{k}\right]=0 \quad, \quad E\left[X_{k} X_{l}^{*}\right]=|X|^{2} \delta_{l, k}, \quad E\left[I_{k}\right]=0
$$

So we get:

$$
\left.E\left[\left|I_{k}\right|^{2}\right] \leq|X|^{2} \sum_{l=-K-k, l \neq 0} E\left[\left|H_{l}\right|^{2}\right\} \frac{1}{N \sin \frac{\pi\left(l+\varepsilon_{\Omega}\right)}{N}}\right)^{2}
$$

For an ideal channel, we can write:

$$
\begin{gathered}
E\left[\left.I_{k}\right|^{2}\right] \leq|X|^{2}|H|^{2} \sum_{l=-K-k, l \neq 0} E\left[\left|H_{l}\right|^{2}\left(\frac{1}{N \sin \frac{\pi\left(l+\varepsilon_{\Omega}\right)}{N}}\right)^{2}\right. \\
E\left[\left|I_{k}\right|^{2}\right]=\sigma_{\varepsilon_{\Omega}}^{2}\left(E_{s}, K, k\right)
\end{gathered}
$$

Numerically, it was determined in [37] that the sum shown in equation (50) is increased by 0.5947 for this case can be rewritten as:

$$
\begin{gathered}
E\left\lfloor\left|I_{k}\right|^{2}\right] \leq 0.5974|X|^{2}|H|^{2}\left(\sin \pi \varepsilon_{\Omega}\right)^{2}, \quad|\varepsilon| \leq 0.5 \\
S N R \geq \frac{|X|^{2}|H|^{2}\left(\frac{\sin \pi \varepsilon_{\Omega}}{\pi \varepsilon_{\Omega}}\right)^{2}}{0.5974|X|^{2}|H|^{2}\left(\sin \pi \varepsilon_{\Omega}\right)^{2}+N_{0}} \\
S N R=\frac{E_{s}\left(\frac{\sin \pi \varepsilon_{\Omega}}{\pi \varepsilon_{\Omega}}\right)^{2}}{N_{0}\left(1+0.5974 \cdot \frac{E_{c}}{N_{0}}\left(\sin \pi \varepsilon_{\Omega}\right)^{2}\right.}
\end{gathered}
$$

$E_{s}=|X|^{2}|H|^{2}$ : is the average energy received. Equation (54) gives directly 
International Journal of Wireless \& Mobile Networks (IJWMN) Vol. 3, No. 4, August 2011

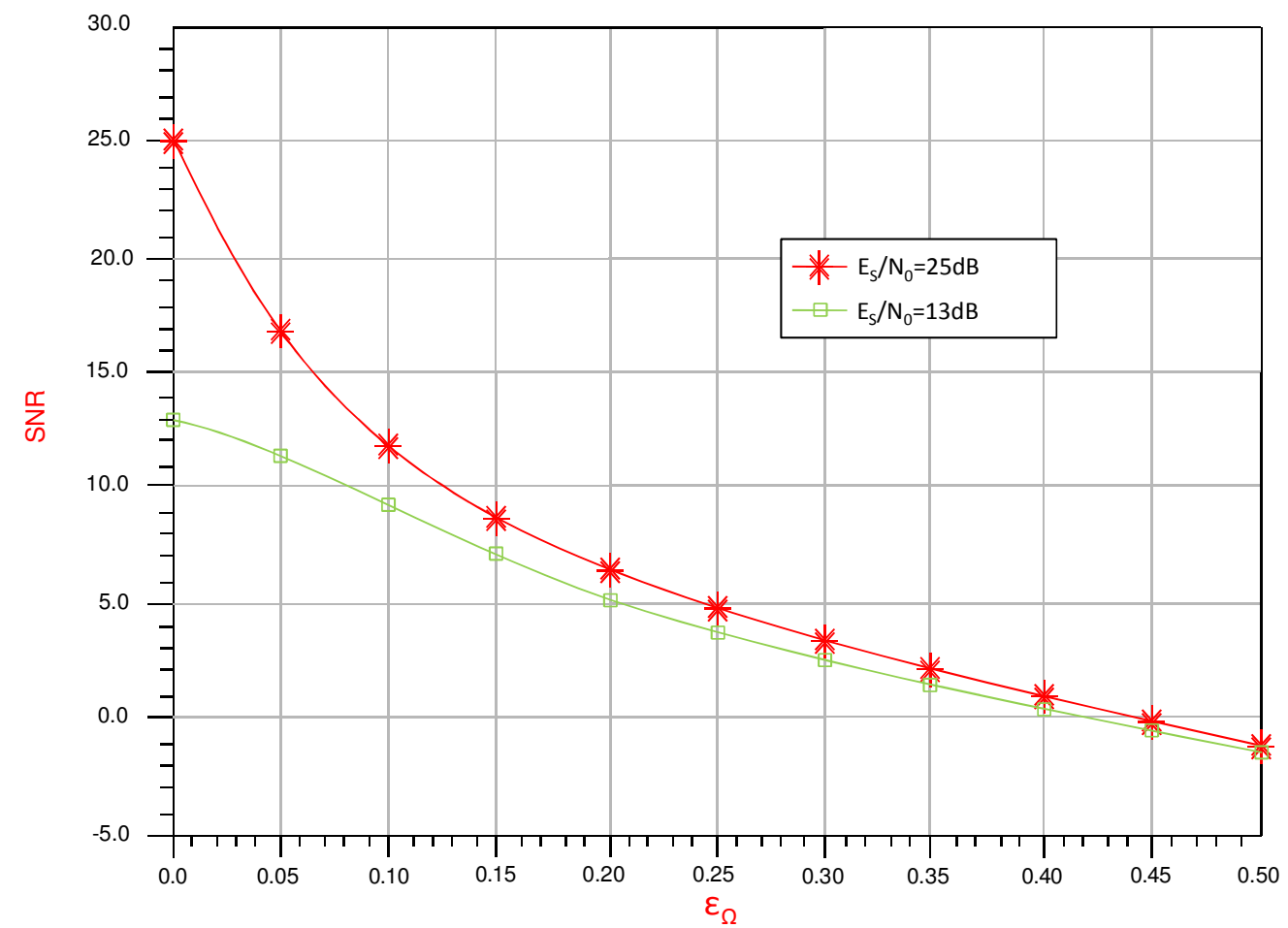

Figure 3. Degradation of signal to noise ratio due to frequency offset, for $\mathrm{N}=256, \mathrm{~K}=96$ for two values of $13 \mathrm{~dB}$ and $\mathrm{ES} / \mathrm{N} 0$ be $25 \mathrm{~dB}$.

\section{CONCLUSION}

In this paper we presented several approaches for time and frequency synchronization on the OFDM transmission systems which can be applied on wireless communication systems such as HiperLAN WLAN. First, studies that we conducted on the impact of errors synchronization on system performance have shown that stress recovery sampling frequency were particularly harsh because of the use of a number Minimum pilot symbols and a maximum number of states constellations. The mathematical approach that we followed during this chapter provides a joint estimate of the channel response and frequency shift of the clock which is an original contribution. We conducted parallel to mathematical analysis, simulations to evaluate relative performance approaches presented in terms of estimators of the frequency approach, the results obtained allow devalue the performance of the system to synchronization effects.

\section{REFERENCES}

[1] U. Tureli, D. Kivanc, and H. Liu, "Experimental and analytical studies on a high resolution OFDM carrier frequency offset estimator, "IEEE Trans. Vehic. Technol, vol. 50, no. 2, pp. 629643, March 2001.

[2] M. Mourad, H. Salem, and B. Ridha, "Analysis of frequency offsets and phase noise effects on an OFDM 802.11 g transceiver," in Proceedings of the 4th International Workshop on Wearable and Implantable Body Sensor Networks (BSN '07), vol. 13, Aachen, Germany, March 2007.

[3] R. Nogueroles, M. Bossert, A. Donder, andV. Zyablov, "Improved performance of a random OFDM A mobile communication system," in IEEE 48th Vehicular Technology Conference (VTC'98), Ottawa, Canada, May18-21, 1998, vol.3, pp. 2502-2506. 
International Journal of Wireless \& Mobile Networks (IJWMN) Vol. 3, No. 4, August 2011

[4] Van der Perre L, Thoen S, Vandenameele P, "Adaptive loading strategy for a high speed OFDMbased WLAN", IEEE Globecom ‘98, Sydney, Australie, November 1998.

[5] http://eesof.tm.agilent.com

[6] S. K. Bassam, "Frequency Synchronization Errors and Effects of Phase Noise on OFDM Systems,”EE359-Wireless Communications Class Projects Stanford University, 2004.

[7] Huang L. C. Tjhung T. T Lu J. "On the optimum number of OFDM subcarriers in fast Rayleigh fading channels". Journal of communication and networks (J. commun. netw.) ISSN 1229-2370. 2001, vol. 3, no2, pp. 142-147.

[8] G. Pujolle, Les Réseaux, Eyrolles, Paris, France, 4th edition, 2000.

[9] G. Bhatia V. Kumar “Adapting MAC 802.11 for performance optimisation of manet using cross layer interaction” International Journal of Wireless \& Mobile Networks (IJWMN) Vol.2, No.4, November 2010

[11] T. Oh, Y.B Choi, J Ryoo, K Stokes "Security management in Wireless Sensor Networks for healthcare”. International Journal of Mobile Communications 2011 - Vol. 9, No.2 pp. 187 - 207

[12] H. Salem, "Contribution à la modélisation des câbles monotones par éléments finis," thesis report ENIT, January 2000.

[13] S. Lim, C. Yu, C. R. Das "A realistic mobility model for wireless networks of scale-free node connectivity”. International Journal of Mobile Communications. Volume 8 - Issue 3 - 2010.

[14] A. R. S. Bahai and B. R. Saltzberg, Multi-Carrier Digital Communications. Theory and Applications of OFDM, Kluwer Academic Publishers/Plenum, New York, NY, USA, 1999.

[15] Shia-Sheng P, "WLAN IEEE802.11a Transceiver, Algorithm, Architecture and Simulation Results", Report of research, November 2001.

[16] V. S. Abhayawardhana and I. J. Wassell, "Common phase error correction for OFDM in wireless communication," in Proceedings of the 3rd International Symposium on Communication Systems, Networks and Digital Signal Processing (CSNDSP '02), Staffordshire, UK, July 2002.

[17] G.L. Stuber, J.R. Barry, S. W. McLaughlin, Y. Li, M.A.Ingram, and T.G. Pratt, "Broadband MIMO-OFDM wireless communications, "Proceedings of the IEEE, vol. 92, no. 2, pp. 271294,February 2004.

[18] D. Petrovic, W. Rave, and G. Fettweis, "Phase noise suppression in OFDM using a Kalman filter," in Proceedings of the 6th International Symposium on Wireless Personal Multimedia Communications (WPMC '03), vol. 3, pp. 375-379, Yokosuka, Japan, October 2003.

[19] D. B. Leeson. A simple model of feedback oscillator noise spectrum. Proc. of the IEEE. pp. 329330, Feb 1966.

\section{Authors}

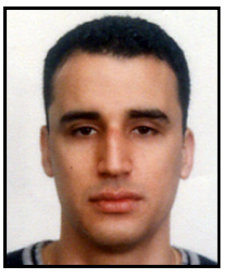

Mourad MELLITI. He received the degree in electrical engineering from National School of Engineering of Tunis, Tunisia, in 2001, and the postgraduate research degree in digital communications, in 2003, from National School of Engineering of Tunis, since 2003 he is a PHD student in the Department of Computer and Communication Technologies. His research interests are mobile communication systems, wireless Transceivers and wireless access network using ADS platforms.

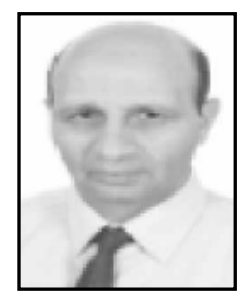

Dr. Salem HASNAOUI is a professor at the Department of Computer and Communication Technologies at the National School of Engineering of Tunis. $\mathrm{He}$ received the Engineer diploma degree in electrical and computer engineering from National School of Engineering of Tunis. He obtained a M.Sc. and third cycle doctorate in electrical engineering, in 1988 and 1993 
International Journal of Wireless \& Mobile Networks (IJWMN) Vol. 3, No. 4, August 2011

respectively. The later is extended to a $\mathrm{PhD}$. degree in telecommunications with a specialization in networks and real-time systems, in 2000. He is author and co-author of more than 40 refereed publications, a patent and a book. His current research interests include real-time systems, sensor networks, QoS control \& networking, adaptive distributed real time middleware and protocols that provide performance assured services in unpredictable environments. $\mathrm{He}$ is the responsible of the research group "Networking and Distributed computing" within the Communications Systems Laboratory at the National School of Engineering of Tunis. He served on many conference committees and journals reviewing processes and he is the designated inventor of the Patent "CAN Inter-Orb protocol- CIOP and a Transport Protocol for Data Distribution Service to be used over CAN, TTP, FlexCAN and FlexRay protocols".

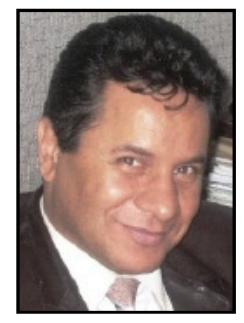

Ridha BOUALLEGUE received the PhD degrees in electronic engineering from the National Engineering School of Tunis. In Mars 2003, he received the HDR degrees in multiuser detection in wireless communications. From September 1990 he was a graduate Professor in the higher school of communications of Tunis (SUP'COM), he has taught courses in communications and electronics. From 2005 to 2008, he was the Director of the National engineering school of Sousse. In 2006, he was a member of the national committee of science technology. Since 2005, he was the laboratory research in telecommunication Director's at SUP'COM. From 2005, he served as a member of the scientific committee of validation of thesis and Hd.R in the higher engineering school of Tunis. His current research interests include wireless and mobile communications, OFDM, space-time processing for wireless systems, multiuser detection, wireless multimedia communications, and CDMA systems. 